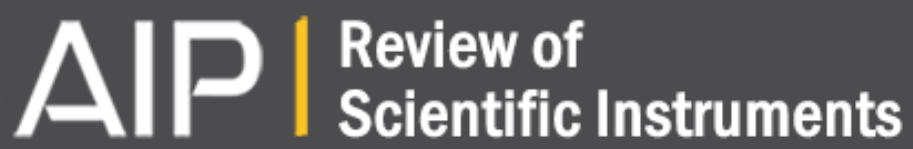

\section{Subresolution axial distance measurements in far-field fluorescence microscopy with precision of 1 nanometer}

Michael Schmidt, Matthias Nagorni, and Stefan W. Hell

Citation: Review of Scientific Instruments 71, 2742 (2000); doi: 10.1063/1.1150685

View online: http://dx.doi.org/10.1063/1.1150685

View Table of Contents: http://scitation.aip.org/content/aip/journal/rsi/71/7?ver=pdfcov

Published by the AIP Publishing

\section{Articles you may be interested in}

Cannula-based computational fluorescence microscopy

Appl. Phys. Lett. 106, 261111 (2015); 10.1063/1.4923402

Multimode waveguide evanescent field fluorescence microscopy: Measurement of cell-substratum separation distance

Appl. Phys. Lett. 94, 033503 (2009); 10.1063/1.3062977

Waveguide evanescent field fluorescence microscopy: Thin film fluorescence intensities and its application in cell biology

Appl. Phys. Lett. 92, 233503 (2008); 10.1063/1.2937840

Nanometer scale marker for fluorescent microscopy

Rev. Sci. Instrum. 76, 073701 (2005); 10.1063/1.1946627

Fluorescence lifetime three-dimensional microscopy with picosecond precision using a multifocal multiphoton microscope

Appl. Phys. Lett. 73, 1769 (1998); 10.1063/1.122276

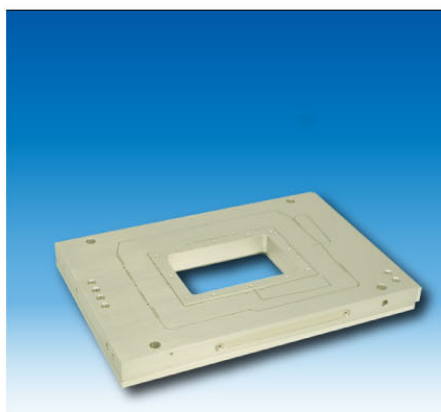

Nanopositioning Systems

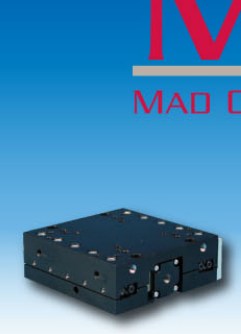

Micropositioning

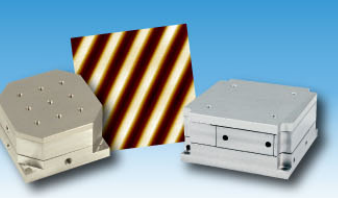

AFM \& SPM

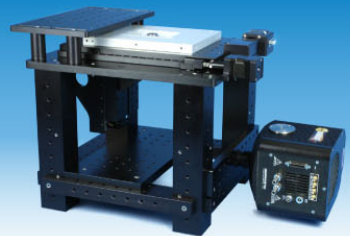

Single molecule imaging 


\title{
Subresolution axial distance measurements in far-field fluorescence microscopy with precision of 1 nanometer
}

\author{
Michael Schmidt, Matthias Nagorni, and Stefan W. Hell ${ }^{\text {a) }}$ \\ High Resolution Optical Microscopy Group, Max-Planck-Institute for Biophysical Chemistry, \\ D-37070 Göttingen, Germany
}

(Received 17 January 2000; accepted for publication 7 April 2000)

\begin{abstract}
Fluorescent objects closer than the diffraction resolution limit can be distinguished in far-field microscopy provided they feature different emission spectra. Utilizing the superior axial resolution of 4Pi-confocal microscopy of 100-150 nm, we investigate the precision with which fluorescence objects with subdiffraction axial distance can be measured in the far field. At a wavelength of 820 $\mathrm{nm}$ distances on the order of $60 \mathrm{~nm}$ between beads and a monomolecular Langmuir-Blodgett layer were determined with a precision of $1.2 \mathrm{~nm}$ within $3.2 \mathrm{~s}$. The reduced spatial extent of the 4 Pi-confocal point-spread-function improves the precision of colocalization measurements in double stained specimens and opens up the prospect on far-field fluorescence profilometry with (sub) nanometer height resolution. (c) 2000 American Institute of Physics.
\end{abstract}

[S0034-6748(00)04007-7]

\section{INTRODUCTION}

In many microscopy applications it is important to measure the distance between fluorescent objects with high precision. Examples are the chromosomal organization in cell nuclei, ${ }^{1}$ as well as colocalization studies of proteins and organelles. The targets of interest are usually labeled with fluorescent markers whose positions are measured in the image. The obtainable precision depends on the signal-to-noise ratio, the microscope spatial resolution, and the effect of chromatic aberrations.

For distance measurements far-field microscopy is often preferable over near-field optics because it is able to image the interior of objects. If the fluorescent markers feature different spectral properties, such as the excitation and emission spectrum, lifetime, or emission polarization, the position of two adjacent objects can be measured separately. It is common knowledge that in this case subdiffraction distances can be quantified. ${ }^{2}$ The diffraction resolution limit does not apply, simply because the objects are resolved by definition.

Recently, distance and localization measurements of distinctly featured fluorescence objects have been reported with a precision of 20-60 $\mathrm{nm}$ using confocal microscopy. ${ }^{1,3,4}$ This method is particularly suitable for multicolor distance and colocalization studies because the confocal point spread function (PSF) is confined in three dimensions (3D) with a typical full width at half-maximum (FWHM) of 150-250 and $500-800 \mathrm{~nm}$ in the lateral and axial direction, respectively. The poorer axial resolution inevitably compromises the precision of axial distance measurements. Due to the coherent use of focused wave fronts of two opposing lenses, 4Pi-confocal fluorescence microscopy provides a 3.5-4-fold improved axial resolution. ${ }^{5}$ With a $4 \mathrm{Pi}$-confocal microscope

\footnotetext{
a) Author to whom correspondence should be addressed; electronic mail: shell@gwdg.de
}

a substantial improvement of axial distance measurements can be expected.

Here we report axial distance measurements with two color channel 4Pi-confocal microscopy with precision of the order of $1 \mathrm{~nm}$. Using a joint two-photon excitation wavelength of $\lambda_{\mathrm{exc}}=820 \mathrm{~nm}$, subresolution distances between fluorescent objects are measured with a precision of about a thousandth of the wavelength. This precision is exemplified by imaging fluorescent beads adhered to a monomolecular Langmuir-Blodgett (LB) layer of fluorescent polydiacetylenes. ${ }^{6}$ It was obtained at a total exposure time of 3-4 s, at an average photon count rate of 7000/s and without exploiting all emissions which could have been obtained before sample bleaching. The two-color 4Pi-confocal recording yields impressive 3D images.

\section{MATERIALS AND METHOD}

Figure 1 sketches the employed 4Pi-confocal microscope of type A. The two detection channels, "yellow" and "green" are defined by a dichroic beamsplitter (DC2) featuring an edge at $570 \mathrm{~nm}$. Residual excitation light of the mode-locked Ti-sapphire laser (Coherent Mira 900F, Santa Clara, CA) was filtered out by a dichroic mirror (DC1) and a 2-mm-thick BG39 glass (Schott, Mainz, Germany). Additional filters are used for separation: whereas the "yellow" channel uses a long pass color glass OG 550 (Schott), the "green" channel further employs a bandpass centered at 525 $\mathrm{nm}$ with a FWHM of $50 \mathrm{~nm}$. The fluorescence was detected with photon-counting avalanche photodiodes with 50\%-70\% quantum efficiency (EG\&G, Canada). The piezo mirror enabled phase adjustment and the charge-coupled device camera facilitated alignment control.

The polydiacetylene LB layer consisted of polymerized dimethyl-bis[pentacosadiinoic-oxyethyl] ammonium bromide which exhibits a strong fluorescence parallel to the polymer backbone. Due to its broad emission from the green 


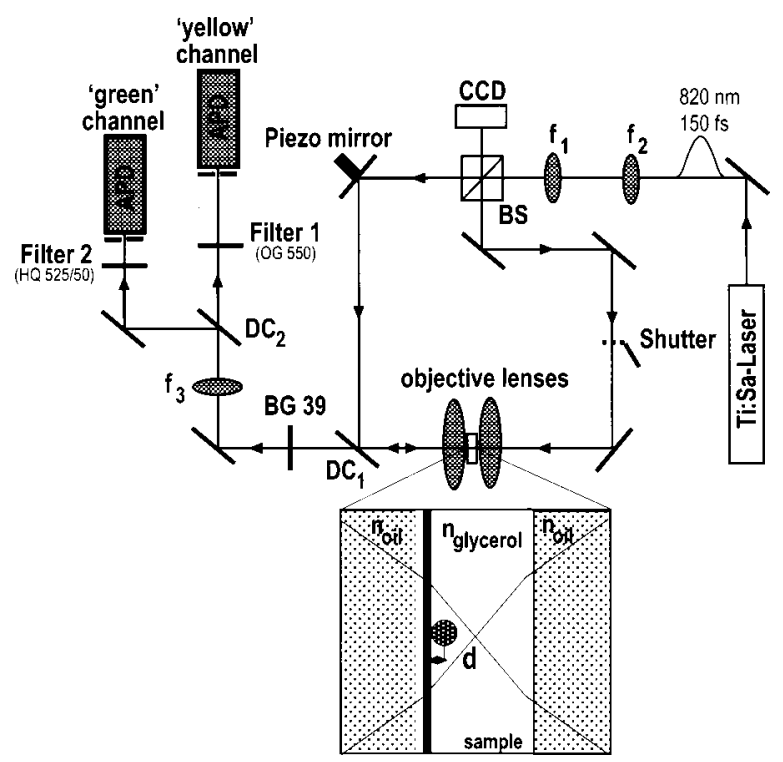

FIG. 1. 4Pi-confocal fluorescence microscope with two-photon excitation and two color channels. The inset sketches a bead adhered to a LB layer covering one of the cover slips. The pulse train of a mode-locked Ti:sapphire laser is split at the beam-splitter (BS) in two coherent parts.

to red spectral range the fluorescence of the LB layer is present in both channels; $23.4 \pm 0.1 \%$ of the total emission entered the "green" channel. The axial responses to the LB layer (Fig. 2) in each channel feature the maximum and the two lobes that are typical for constructive interference in two-photon excitation 4Pi-confocal microscopy of type A. The axial position of the LB layer was localized by fitting the 4Pi-confocal response with a function consisting of three Gaussian peaks:

$$
\begin{aligned}
I(z)= & I_{0}+I_{\max }\left(\exp \left(-\frac{\left(z-z_{c}\right)^{2}}{2 w^{2}}\right)\right. \\
& +\kappa_{1} \exp \left(-\frac{\left(z-z_{c}+b\right)^{2}}{2 w^{2}}\right) \\
& \left.+\kappa_{2} \exp \left(-\frac{\left(z-z_{c}-b\right)^{2}}{2 w^{2}}\right)\right) .
\end{aligned}
$$

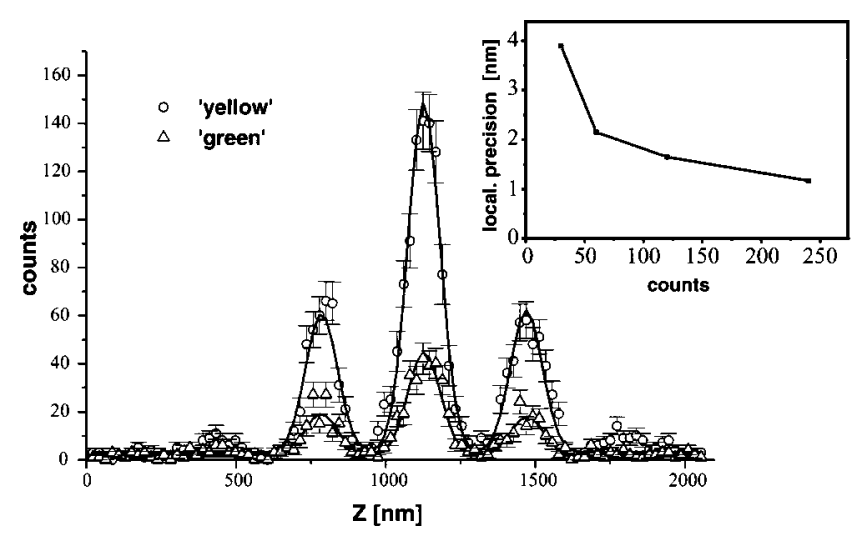

FIG. 2. Axial responses of the monomolecular LB layer in the "green" and "yellow" detection channel of the 4Pi-confocal microscope; The inset shows the standard deviation of localization of the center of the response as a function of the maximal count rate.
A similar fit was used for the bead. The parameter $w$ quantifies the axial width of the peak function, $b$ is the distance between the peaks, and $\kappa_{1}, \kappa_{2}$ are their relative heights. $z_{c}$ is the desired axial position of the center of the response. $I_{0}$ is a constant background in the image. The $\chi^{2}$ fit was realized using the Levenberg-Marquardt algorithm.

\section{RESULTS}

For a given PSF the localization precision is determined by the signal-to-noise ratio (SNR) alone. As the SNR is described by Poisson statistics, we first investigated the precision that can be anticipated for a given number of counts per pixel under Poisson noise conditions. For this purpose we evaluated theoretical 4Pi-confocal responses and extracted the precision with which we could determine the center for typical SNR encountered in our experiment (Fig. 2, inset). We found that for 100-200 counts per $20 \mathrm{~nm}$ pixel in $Z$ direction we should obtain a precision of the order of $1 \mathrm{~nm}$.

Potential obstacles are longitudinal chromatic aberrations. Two-photon excitation at $820 \mathrm{~nm}$ allowed the excitation of both fluorophores in the same volume. Therefore on the excitation side chromatic aberration cannot occur. However, chromatic aberration associated with the two detection colors leads to an axial offset of the detection PSFs defined by the pinholes. This offset can be eliminated by shifting one of the pinholes along the optic axis. Residual chromatic aberration manifests itself as an apparent axial shift of the center of the response, but also as a difference in shape between the responses in each channel of the (same) LB layer. The latter effect is due to the fact that the relative height of the two lobes of the $4 \mathrm{Pi}$-confocal PSF strongly depends on the relative axial position of the excitation and detection foci. Given that the lobes in one channel have equal height, longitudinal chromatic aberration would express itself as a different lobe height in the other channel. Therefore the side lobes are a powerful tool to accurately correct for chromatic aberration.

After correction, a total of 100 axial scans revealed an offset of $0.4 \pm 0.3 \mathrm{~nm}$ so that chromatic aberrations could be safely neglected in the following measurements. The standard deviation of the difference between the center positions of the axial intensity profiles of each channel was 3.8 $\pm 0.3 \mathrm{~nm}$. The medium peak count number of the axial line scan in the "yellow" and "green" channel was 134 and 43, respectively. Based on the Poisson statistics model of Fig. 2 (inset) we expect a standard deviation of $3.6 \mathrm{~nm}$, which is in agreement with the measured value. This indicates that the precision was limited only by photon statistics.

Next we recorded stacks of $X Z$ images of fluorescent beads (Yellow-Green Fluospheres; Molecular Probes, Eugene, OR) on the LB layer in the 4Pi-confocal mode (Fig. 3). The diameter of the beads was specified to $110 \mathrm{~nm}$; the maximum emission was at a wavelength of $515 \mathrm{~nm}$. A confocal recording was also taken for comparison (data not shown). The $X Y Z$-image size was $0.8 \times 0.8 \times 2.2 \mu \mathrm{m}^{3}$ with a pixelation of 40,40 , and $23.3 \mathrm{~nm}$ in the $x, y$, and $z$ directions, respectively. The pixel dwell time was $2 \mathrm{~ms}$. The bead was embedded in glycerol whose refractive index $(n=1.47)$ differed from that of the oil $(n=1.52)$ and the cover slip (Fig. 
(a)

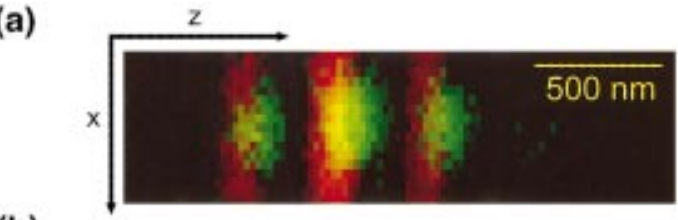

(b)

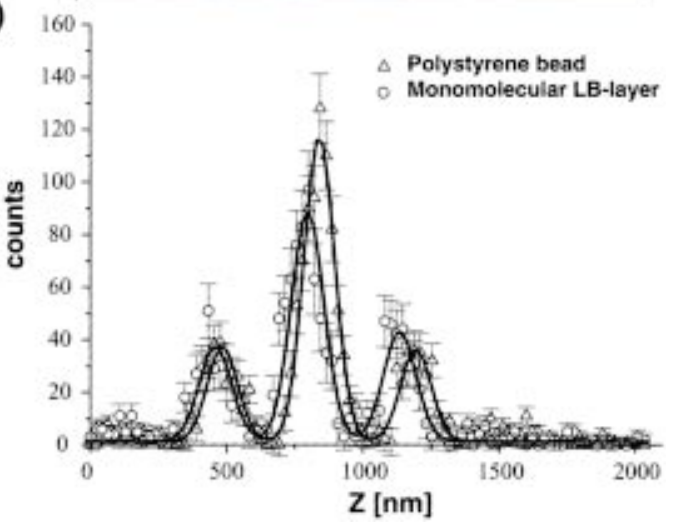

FIG. 3. (Color) (a) Raw data of a two-color 4Pi-confocal XZ image of a 110 -nm-diam fluorescent bead on a fluorescent monomolecular layer. The green and red colors indicate the two color channels. (b) Axial profile $I(z)$ through the bead center for both channels.

1). The concomitant change of the scale along the optic axis by a factor of $\gamma_{\text {refr }}=0.93$, which is due to spherical aberrations, was taken into account by scaling. ${ }^{7}$

The axial distance of the two point objects at a lateral coordinate $(x, y)$ is given by $d=\gamma_{\text {refr }}\left(z_{c}^{\mathrm{LB}}\right.$ layer $\left.-z_{c}^{\text {bead }}\right)$, with $z_{c}^{\text {bead }}, z_{c}^{\mathrm{LB}}$ layer denoting the measured position for the bead and the LB layer, respectively. $16 \pm 0.1 \%$ of the fluorescence of the bead entered the "yellow" channel. Therefore the axial profiles $I^{\mathrm{LB}} \operatorname{layer}(z)$ and $I^{\text {bead }}(z)$ were corrected for this cross-talk by a weighted subtraction. The axial positions $z_{c}^{\mathrm{LB}}$ layer,$z_{c}^{\text {bead }}$ and their standard deviations were determined by fitting $I^{\mathrm{LB}}$ layer $(z)$ and $I^{\text {bead }}(z)$ with Eq. (1) in each channel. To increase the precision, all the profiles whose maximum was at least $75 \%$ of the global intensity maximum of the bead image, 16 in total, were considered. Since the data stack is three dimensional we could also rely on profiles from neighboring $X Z$ sections. In the experiment shown in Fig. 3 we measured $d=62 \pm 1.2 \mathrm{~nm}$.

\section{DISCUSSION}

It is interesting to note that $d$ is 2.5 times below the axial diffraction limit of $140 \mathrm{~nm}$ of the $4 \mathrm{Pi}$-confocal microscope and 10 times below that of a single lens two-photon confocal microscope. ${ }^{8}$ The recording time of the measured area was only 3.2 s. The total number of counts was 21266 and 23288 for the bead and the LB layer, respectively. Given that an average fluorophore can emit about $5 \times 10^{4}$ photons of which $2 \%$ are detected, ${ }^{9}$ each molecule can contribute with $\sim 10^{3}$ photons. Therefore, 20-25 fluorophore molecules are sufficient to establish distances between fluorophore agglomerations with a precision of $1 \mathrm{~nm}$. Stable fluorophores can emit up to $10^{6}$ photons, in which case a precision of 1 $\mathrm{nm}$ is achieved already with single molecules. However, this calculation strictly applies only if the bleaching of the two markers occurs at the same rate. Otherwise, the necessary

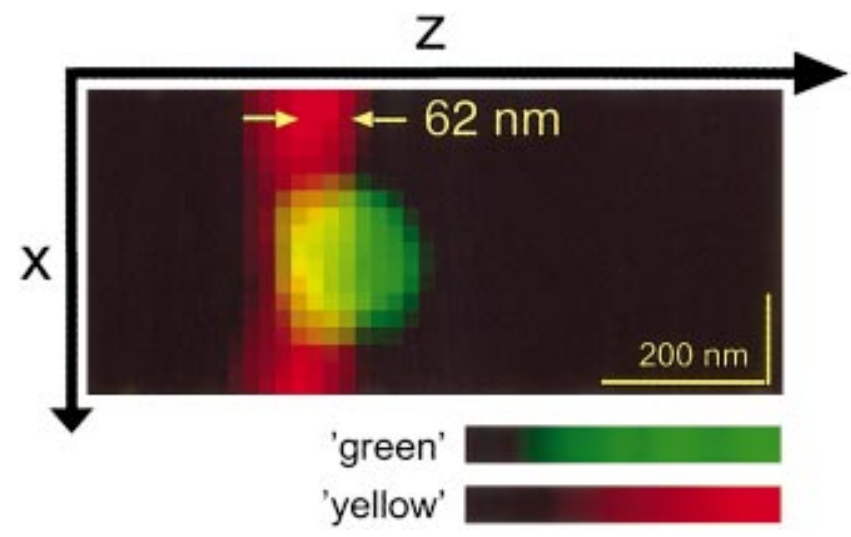

FIG. 4. (Color) 4Pi-confocal $X Z$ image of a two-color-channel data stack displays the fluorescent bead on the LB layer. Note the subwavelength dimensions in the image.

cross-talk correction by weighted subtraction would not be applicable, unless the bleaching is considered as a function of time. In the present experiment, we kept the excitation intensity low $(\sim 200 \mu \mathrm{W}$ of time-averaged power) to avoid bleaching. As our system works close to the Poisson noise limit, we expect that the precision can be improved to the sub-nm regime, simply by collecting a higher number of photons.

If at all present, Förster resonance energy transfer $(\text { FRET })^{10}$ between the layer and the fluorophore in the bead would be restricted to the area of immediate contact. Being an induced dipole interaction FRET would only affect the fluorescence molecules at the bead surface and up to a maximum depth of $\sim 10 \mathrm{~nm}$. Since the vast majority of the molecules in the bead are too remote from the LB layer, we do not expect FRET to compromise the localization of the bead. The LB layer might be slightly quenched by FRET where it touches the bead (Fig. 3), however, the fluorescence from the layer is strong enough to reveal its precise localization. FRET is undesired in our case, however, we note for completeness that FRET is an established nonimaging technique for measuring distances between fluorophore agglomerations or single fluorophores ${ }^{11}$ in the small range of $1-10 \mathrm{~nm}$, which works well if the parameters of the induced dipole interaction are known.

Finally we show that the axial side lobes in the 4Piconfocal image data is readily removed by a linear mathematical filter taking into account the relative height and position of the lobes. The filter is applied to each channel and eliminates the interference effects. The result is an unambiguous representation of the object in the image. Figure 4 impressively demonstrates the ability of the 4Pi-confocal microscope to vividly visualize the bead on the layer and the distance between them.

By using both coherent illumination and detection in 4 Pi-confocal microscopy (type $\mathrm{C})^{12}$ the axial extent of the main maximum can be further reduced, and a total resolution improvement by a factor of 7 achieved. Therefore type $\mathrm{C}$ 4Pi-confocal microscopy has the potential to further enhance the precision.

In conclusion, we have demonstrated fast subresolution axial distance measurements in far-field fluorescence micros- 
copy with a precision of $1.2 \mathrm{~nm}$, corresponding to almost a thousandth of the wavelength. The precision can be further augmented by either increasing the number of registered photons or by further reducing the spatial extent of the PSF. Both measures should improve the precision to the picometer scale.

\section{ACKNOWLEDGMENT}

This work was supported by a grant from the Deutsche Forschungsgemeinschaft (DFG, Bonn).

${ }^{1}$ H. Bornfleth, K. Satzler, R. Eils, and C. Cremer, J. Microsc. 189, 118 (1998).
${ }^{2}$ D. H. Burns, G. D. Callis, G. D. Christian, and E. R. Davidson, Appl. Opt. 24, 154 (1985).

${ }^{3}$ M. v. Oijen, J. Köhler, J. Schmidt, M. Müller, and G. J. Brakenhoff, Chem. Phys. Lett. 292, 183 (1998).

${ }^{4}$ E. M. M. Manders, J. Microsc. 185, 321 (1997).

${ }^{5}$ S. W. Hell, M. Schrader, and H. T. M. van der Voort, J. Microsc. 185, 1 (1997).

${ }^{6}$ M. Schrader, U. G. Hofmann, and S. W. Hell, J. Microsc. 191, 135 (1998).

${ }^{7}$ A. Egner, M. Schrader, and S. W. Hell, Opt. Commun. 153, 211 (1998).

${ }^{8}$ M. Nagorni and S. W. Hell, J. Struct. Biol. 123, 236 (1998).

${ }^{9}$ J. Pawley, Handbook of Biological Confocal Microscopy (Plenum, New York, 1995).

${ }^{10}$ T. Förster, Ann. Phys. (Leipzig) 2, 55 (1948).

${ }^{11}$ S. Weiss, Science 283, 1676 (1999).

${ }^{12}$ S. Hell and E. H. K. Stelzer, J. Opt. Soc. Am. A 9, 2159 (1992). 